\title{
Supplemental vitamin D enhances the recovery in peak isometric force shortly after intense exercise
}

\author{
Tyler Barker ${ }^{1 *}$, Erik D Schneider ${ }^{2}$, Brian M Dixon², Vanessa T Henriksen ${ }^{1}$ and Lindell K Weaver ${ }^{3,4}$
}

\begin{abstract}
Background: Serum 25-hydroxyvitamin D $(25(\mathrm{OH}) \mathrm{D})$ concentrations associate with skeletal muscle weakness (i.e., deficit in skeletal muscle strength) after muscular injury or damage. Although supplemental vitamin D increases serum $25(\mathrm{OH}) \mathrm{D}$ concentrations, it is unknown if supplemental vitamin $\mathrm{D}$ enhances strength recovery after a damaging event.

Methods: Reportedly healthy and modestly active (30 minute of continuous physical activity at least 3 time/week) adult males were randomly assigned to a placebo $\left(n=13\right.$, age, 31(5) y; BMl, 26.9(4.2) $\mathrm{kg} / \mathrm{m}^{2}$; serum 25(OH)D, 31.0(8.2) $\mathrm{ng} / \mathrm{mL}$ ) or vitamin D (cholecalciferol, $4000 \mathrm{IU} ; \mathrm{n}=15$; age, 30(6) y; BMl, 27.6(6.0) kg/m²; serum 25(OH)D, 30.5(9.4) ng/mL) supplement. Supplements were taken daily for $35-\mathrm{d}$. After $28-\mathrm{d}$ of supplementation, one randomly selected leg performed an exercise protocol (10 sets of 10 repetitive eccentric-concentric jumps on a custom horizontal plyo-press at $75 \%$ of body mass with a 20 second rest between sets) intended to induce muscle damage. During the exercise protocol, subjects were allowed to perform presses if they were unable to complete two successive jumps. Circulating chemistries (25(OH)D and alanine (ALT) and aspartate (AST) aminotransferases), single-leg peak isometric force, and muscle soreness were measured before supplementation. Circulating chemistries, single-leg peak isometric force, and muscle soreness were also measured before (immediately) and after (immediately, 1-h [blood draw only], 24-h, 48-h, 72-h, and 168-h) the damaging event.
\end{abstract}

Results: Supplemental vitamin D increased serum $25(\mathrm{OH})$ D concentrations $(P<0.05 ; \approx 70 \%)$ and enhanced the recovery in peak isometric force after the damaging event $(P<0.05 ; \approx 8 \%$ at $24-h)$. Supplemental vitamin $D$ attenuated $(P<0.05)$ the immediate and delayed (48-h, 72-h, or 168-h) increase in circulating biomarkers representative of muscle damage (ALT or AST) without ameliorating muscle soreness $(P>0.05)$.

Conclusions: We conclude that supplemental vitamin D may serve as an attractive complementary approach to enhance the recovery of skeletal muscle strength following intense exercise in reportedly active adults with a sufficient vitamin D status prior to supplementation.

Keywords: Vitamin D, Muscle strength, Muscle damage, Exercise

\section{Background}

Vitamin D is a fat-soluble micronutrient receiving considerable clinical and research interest. These interests stem from earlier work identifying the importance of vitamin D on bone health. Since the resolution of rickets with vitamin D fortification in food, and more recently with the polarizing debates regarding the appropriate daily intake of vitamin D and the serum 25-hydroxyvitamin D

\footnotetext{
* Correspondence: tyler.barker@imail.org

${ }^{1}$ The Orthopedic Specialty Hospital, 5848 S. Fashion Blvd., Murray, UT 84107, USA

Full list of author information is available at the end of the article
}

(25(OH)D) concentration demarcating vitamin D sufficiency, the interest in vitamin $\mathrm{D}$ has spread across a range of physiological systems. Of these systems, there is mounting data indicating that vitamin $\mathrm{D}$ is a potent regulator of skeletal muscle physiology. In part, the regulatory interest of vitamin D on skeletal muscle physiology originated from a clinical observation [1] and diverse vitamin D interventions suggesting that increasing serum $25(\mathrm{OH}) \mathrm{D}$ concentrations could improve skeletal muscle strength or function in patients with osteomalacia $[2,3]$.

\section{Biomed Central}

(c) 2013 Barker et al.; licensee BioMed Central Ltd. This is an open access article distributed under the terms of the Creative Commons Attribution License (http://creativecommons.org/licenses/by/2.0), which permits unrestricted use, distribution, and reproduction in any medium, provided the original work is properly cited. 
Following these clinical findings, cell and experimental animal studies subsequently found that vitamin D regulates calcium handling and transport, phosphate metabolism, cytoskeletal protein expression, and the activation of mitogen activate protein kinase signaling pathways in skeletal muscle [4-11]. The genomic and non-genomic events established at the molecular and cellular levels extended our knowledge pertaining to the governing role of vitamin D in skeletal muscle function [for review see 12,13]. Today, the association between low serum $25(\mathrm{OH}) \mathrm{D}$ concentrations and muscle strength has evolved as results from cell and experimental animal studies are continuously translated to the causative explanation of muscular weakness in patients with low serum $25(\mathrm{OH}) \mathrm{D}$ concentrations.

The association between muscle strength and vitamin $\mathrm{D}$ has led to the thesis that increasing serum $25(\mathrm{OH}) \mathrm{D}$ concentrations could be a complementary approach to enhance strength (skeletal muscle) following intense exercise that induces immediate and persistent deficits in muscular strength. Consistent with this postulate, we found a positive correlation between serum 25(OH)D concentrations and strength recovery following muscular injury in subjects who were not supplementing with vitamin D [14]. Extending these findings, vitamin D treatment restored muscular strength after a crush injury in experimental rats [15]. The regenerative influence of vitamin D on skeletal muscle was attributed to the increased proliferation and decreased apoptosis of muscle cell nuclei and not to a robust alteration in satellite cell number or tissue-infiltrating leukocytes [15]. Although the results from experimental rats establish a causative influence of vitamin $\mathrm{D}$ on muscle regeneration after insult [15], in humans, data is less conclusive as results are limited to the association between vitamin D and strength $[14,16]$.

The purpose of this study was, therefore, to identify the influence of supplemental vitamin $\mathrm{D}$ on strength recovery following intense exercise that induces a persistent (i.e., 24-h to 72-h post) deficit in peak isometric force in humans. We hypothesized that supplemental vitamin D enhances strength recovery after a damaging event. Subjects were randomly assigned to a daily placebo or vitamin D (cholecalciferol, $4000 \mathrm{IU}$ ) supplement in a double-blind manner. To induce persistent muscular weakness, each subject performed an intense, unilateral-leg exercise protocol consisting of repetitive eccentric-concentric contractions. In addition to monitoring single-leg muscle strength, we evaluated circulating proteins and the delayed onset of muscle soreness to assess the presence of muscle damage.

\section{Methods}

The Urban Central Region Institutional Review Board at Intermountain Healthcare (Salt Lake City, UT, USA) approved this study. Subjects were informed of and provided written and verbal consent to the experimental protocol and procedures. Reportedly healthy and modestlyactive (i.e., $30 \mathrm{~min}$ of continuous physically active at least 3 times per week) males were recruited to participate in this study. Potential subjects were excluding if they were taking any dietary supplements, using any prescribed or recommended medications, reported known history of any disease or condition requiring medical attention, suffered a lower leg injury during the previous year that required the use of crutches, planning on increasing or decreasing the amount of time spent in the sun or tanning bed, or traveling south of $37^{\circ} \mathrm{N}$ in latitude during study participation. Subjects were also excluded if they were morbidly obese (body mass index $(\mathrm{BMI})>40 \mathrm{~kg} / \mathrm{m}^{2}$ ) or displayed strength or power output asymmetry (i.e., > 5\% difference in peak isometric or power output between legs). Symmetry in leg strength was important since all strength testing and the exercise protocol (see below) were performed unilaterally and statistical comparisons were made within and between legs. Data was collected during the winter (between November and March) in Salt Lake City, UT, USA ( $40^{\circ} \mathrm{N}$ latitude).

\section{Study design and protocol}

This study consisted of a randomized, double-blind, placebo-controlled experimental design. Subjects were randomly assigned to one of two groups: (1) placebo $(\mathrm{n}=13)$ or (2) vitamin D (cholecalciferol, $4000 \mathrm{IU} ; \mathrm{n}=15$ ) supplementation. Supplements (donated from USANA Health Sciences, Inc.) were taken daily for 35-d. During participation, subjects were asked to keep their diet consistent with their regular eating habits during the previous year and to refrain from using any dietary supplements. Subjects were also asked to refrain from physical activity, and using aspirin, ibuprofen, naproxen sodium, acetaminophen, or other anti-inflammatory agents 72-h prior to a blood draw.

Each subject provided eight fasting blood samples: (1) baseline, (Bsl and 28-d before the exercise protocol), (2) immediately before [Pre] and (3) immediately [Post], (4) 1-h, (5) 24-h, (6) 48-h, (7) 72-h, and (8) 168-h after the exercise protocol. Single-leg strength testing and perceived muscle soreness were performed on seven different occasions: (1) Bsl, (2) Pre, (3) Post, (4) 24-h, (5) 48-h, (6) 72-h, and (7) 168-h. Each visit commenced within 1-h of waking and between 06:00 and 09:00 a.m. The time remained consistent within each subject across all visits. Perceived muscle soreness was performed before the blood draw procedure, and the blood draw was performed prior to strength testing. Supplementation started at Bsl after the blood draw, perceived muscle soreness, and single-leg strength testing procedures. 


\section{Exercise induced muscle damage}

Each subject had one randomly selected leg (stretchshortening contraction; SSC) perform an intense exercise protocol. The other leg served as the contralateral control $(\mathrm{CON})$. The exercise protocol was performed 28-d after Bsl and consisted of 10 sets of 10 repetitive eccentricconcentric jumps at $75 \%$ of body mass with a $20 \mathrm{sec}$ rest between each set, as described elsewhere [14,17]. Briefly, subjects were instructed and verbally encouraged to perform each set with maximal effort and to jump as-high-as possible through a full range of motion $\left(90^{\circ}\right.$ of knee flexion-to-full extension). If subjects were no longer able to complete two-successive jumps, subjects were then allowed to perform presses (foot remaining on the force plate) through a full range of motion $\left(90^{\circ}\right.$ of knee flexionto-full extension). If subjects were unable to complete two successive presses, the exercise protocol was terminated. The rationale for including the presses was to allow the subjects to successfully complete the requested number of sets and repetitions. However, switching from jumps to presses presumably lowered the eccentric magnitude during loading and some subjects were unable to complete the protocol.

The mean number of jumps and presses completed during the exercise protocol were not significantly (statistically) different between the placebo (jumps, $62 \pm 9$; presses $19 \pm 4$ ) and vitamin D (jumps, $59 \pm 8$; presses, $30 \pm 6)$ groups. Six $(46 \%)$ of the placebo subjects and 9 (60\%) of the vitamin D subjects completed the exercise protocol. Blood chemistries, perceived muscle soreness, and leg strength data were not significantly different between those who finished and those who did not finish the exercise protocol or between the placebo and vitamin D groups as determined by a repeated measures analysis of variance (ANOVA).

\section{Blood sample handling}

Fasting blood samples were obtained from the antecubital vein into one $4.0 \mathrm{~mL}$ purple-top Becton Dickinson (BD; Franklin Lakes, NJ, USA) Vacutainer tube (K2 EDTA $7.2 \mathrm{mg}$ plasma), one $4.5 \mathrm{~mL}$ green-top BD Vacutainer tube (PST Gel and lithium heparin, 83 units), and one $6.0 \mathrm{~mL}$ red-top serum BD Vacutainer tube. Plasma was separated by centrifugation (Heraeus Labofuge 400 series, Buckinghamshire, England) at $2400 \mathrm{~g}$ for $6 \mathrm{~min}$ within 20 min of sample collection. Following separation, plasma samples were sent to ARUP Laboratories (Salt Lake City, UT USA) for clinical chemistries (see below). After coagulation, serum was separated by centrifugation (VWR International, Clinical 50 Centrifuge) at $1100 \mathrm{~g}$ for $20 \mathrm{~min}$, and then aliquoted into several small micro-centrifuge tubes. Aliquoted serum samples were stored at $-80^{\circ} \mathrm{C}$ (Revco Freezer, GC Laboratory Equipment, Asheville, NC, USA) until analysis (see below).

\section{Analytical procedures}

\section{Serum 25(OH)D concentrations}

Serum $25(\mathrm{OH}) \mathrm{D}$ concentrations $(\mathrm{ng} / \mathrm{mL})$ were measured in duplicate (coefficient of variation $(\mathrm{CV})=3.90 \%$ ) in each blood sample, as described previously [18]. Briefly, analytes were separated on an Agilent high performanceliquid chromatography system (series 6460, Model G6460A, Santa Clara, CA, USA) and detected on an Agilent tandem mass spectrometer (Series 6410, Model G6410B, Santa Clara, CA, USA) using atmospheric pressure chemical ionization $(\mathrm{APCI})$ detection $\left(350^{\circ} \mathrm{C}\right.$ gas temperature, $400^{\circ} \mathrm{C}$ vaporizer). The $25(\mathrm{OH}) \mathrm{D}_{3}$, deuterated $25(\mathrm{OH}) \mathrm{D}_{3}$ internal standard, and $25(\mathrm{OH}) \mathrm{D}_{2}$ precursor ions were $383.3,386.3$, and 395.4 , respectively. The $25(\mathrm{OH}) \mathrm{D}_{3}$, deuterated $25(\mathrm{OH}) \mathrm{D}_{3}$, and $25(\mathrm{OH}) \mathrm{D}_{2}$ product ions were 365.3 , 368.3, and 208.9, respectively. Serum $25(\mathrm{OH}) \mathrm{D}_{2}$ and $25(\mathrm{OH}) \mathrm{D}_{3}$ concentrations were determined relative to authentic standards and corrected for recovery of the $25(\mathrm{OH}) \mathrm{D}_{3}$ internal standard. Serum $25(\mathrm{OH}) \mathrm{D}_{2}$ (limit of detection $=2.0 \mathrm{ng} / \mathrm{mL}$ ) was not detected in any of the subjects, and therefore, serum $25(\mathrm{OH}) \mathrm{D}$ total concentrations are referred to as serum $25(\mathrm{OH}) \mathrm{D}$ concentrations hereafter.

\section{Clinical chemistries}

A comprehensive metabolic panel was performed on Bsl plasma samples. Plasma AST (U/L), ALT (U/L), PTH $(\mathrm{pg} / \mathrm{mL})$, and calcium $(\mathrm{mg} / \mathrm{dL})$ concentrations were measured in each blood sample (ARUP Laboratories, Salt Lake City, UT, USA).

\section{Single-leg strength testing}

Single-leg strength testing was performed on a horizontal Plyo-Press (Athletic Republic, Park City, UT, USA), as described previously [17-19]. In brief, the Plyo-Press sled was adjusted for each subject to align the knee and hip joint flexion angles to $90^{\circ}$ with the abdominal, low back region secured and stabilized to the Plyo-Press sled with a harness. Subjects performed submaximal isometric contractions on each leg at increasing intensities $(\approx 50,75$, and $90 \%$ ) prior to performing the testing contractions to become familiar with the testing protocol and procedure. Leg selection (i.e., CON or SSC leg) at the start of each testing session was randomized and followed by an alternating sequence of leg contractions. Peak isometric contractions (i.e., hip and knee extension) on each leg were performed in triplicate $(\mathrm{CON}$ leg $\mathrm{CV}=2.62 \%$; SSC leg $\mathrm{CV}=6.13 \%$ ). Each isometric contraction was $3 \mathrm{sec}$ in duration and separated by $1 \mathrm{~min}$ of rest. Subjects were verbally instructed and strongly encouraged to exert maximal force against the mounted force platform. Peak isometric force was defined as the highest resultant force produced from the three isometric tests and expressed relative to body mass $(\mathrm{N} / \mathrm{kg})$. 
Single-leg peak power output measures followed the single-leg isometric contractions and were measured on the same horizontal Plyo-Press with the same securing procedures described above. Starting from an extended position (full extension $=0^{\circ}$ ), subjects performed several submaximal jumps to become familiar with the testing protocol and procedure. Thereafter, subjects performed repetitive maximal effort single-leg jumps (i.e., hip and knee flexion-extension cycles). Subjects were instructed and verbally encouraged to perform the jumps as fast as possible and through a full range of motion $\left(90^{\circ}\right.$ of knee flexion-to-full extension). Each test was $20 \mathrm{sec}$ in duration with the weight-stack resistance set at $75 \%$ of body mass. The time-aligned product of the resultant force (N) acquired from the force platform and the weightstack velocity $(\mathrm{m} / \mathrm{s})$ data obtained from the displacement transducer were used to calculate power output. Peak power output was defined as the highest power output produced during the $20 \mathrm{sec}$ test for each leg.

Plyo-Press output data were measured from signals obtained from a mounted force plate (Advanced Mechanical Technology, Watertown, MA, USA) and from a displacement transducer (UniMeasure PA-50-NJC, Corvallis, OR, USA) attached to the weight stack. Data were sampled at $200 \mathrm{~Hz}$ with a low-pass filter at $10 \mathrm{~Hz}$ using DartPower software (Athletic Republic, Park City, UT, USA, version 2.0).

\section{Muscle soreness}

To assess muscular soreness, subjects lowered their body into a squat position $\left(90^{\circ}\right.$ of hip and knee flexion) for 5 seconds while resting their back against a wall. This was performed in triplicate at each visit. In the squat position, subjects rated the perceived soreness of their gluteus, quadriceps, hamstrings, and calves in each leg by using a visual analog scale (10 cm in length) of 1 to 10 , with 0 being 'no pain', 10 being the 'worst possible pain', and 5 being 'tender to touch but not to contractions' [20]. Subjects made a perpendicular mark on the visual analog scale from which perceived soreness was quantified by measuring to the nearest tenth of a centimeter.

\section{Statistical analyses}

Data were checked for normality prior to all statistical analyses with a Shapiro-Wilk test. Statistical significance of subject characteristic and exercise protocol data (i.e., number of jump and press repetitions) were assessed using a two-sample $t$-test with a Bonferroni adjustment. Statistical significance of data (i.e., serum 25(OH)D, calcium, PTH, peak isometric force, and peak power output) were assessed using a repeated-measures ANOVA and post hoc t-tests with a Bonferroni correction on multiple pairwise comparisons when appropriate. Due to nonnormally distributed data, statistical significance of ALT,
AST, peak force, and perceived muscle soreness (gluteus, quadriceps, hamstrings, and calves) data were assessed with separate Friedman ANOVA tests. Statistical significance in strength recovery were assessed with a twosample $t$-test (peak power output) with a Bonferroni correction for multiple pairwise comparisons or with a Wilcoxon Signed-Ranked Test (peak isometric force) if data were non-normally distributed. Relationships between variables were assessed with a Pearson productmoment linear correlation. All statistical analyses were performed with SYSTAT (version 13.1, Chicago, IL, USA). Statistical significance was set at $P<0.05$. Data presented as mean (SD) unless noted otherwise.

\section{Results}

Subject characteristics and serum 25(OH)D concentrations. The average serum $25(\mathrm{OH}) \mathrm{D}$ concentration was 30.8 (1.6) ng/mL upon enrollment and prior to randomization. Using the United States Endocrine Society guidelines [21], three, ten, and 15 subjects were categorized as vitamin $\mathrm{D}$ deficient (serum $25(\mathrm{OH}) \mathrm{D}<20 \mathrm{ng} / \mathrm{mL}$ ), vitamin D insufficient (serum 25(OH)D 20 to $29 \mathrm{ng} / \mathrm{mL}$ ), or vitamin D sufficient (serum 25(OH)D > $30 \mathrm{ng} / \mathrm{mL}$ ), respectively. Thus, for the most part, subjects displayed a good vitamin $\mathrm{D}$ level prior to randomization and supplementation.

Following randomization and prior to supplementation (i.e., at Bsl), subject characteristics and serum 25(OH)D concentrations were similar between the groups (Table 1). In the placebo group, six subjects were categorized as vitamin $D$ deficient $(n=1)$ or insufficient $(n=5)$, while the majority of the subjects were vitamin $\mathrm{D}$ sufficient $(n=7)$. Likewise, several subjects were categorized as vitamin $D$ deficient $(n=2)$ or insufficient $(n=5)$, but most subjects were vitamin $D$ sufficient $(n=8)$ in the vitamin $\mathrm{D}$ group prior to supplementation.

At the conclusion of supplementation (i.e., 168-h [or 35 -d total of supplementation]), the number of vitamin $\mathrm{D}$ deficient, insufficient, and sufficient subjects remained constant in the placebo group compared to that at Bsl. Likewise, the serum 25(OH)D concentration did not significantly change in the placebo group from Bsl to 168-h (29.1 (7.9) ng/mL) (Additional file 1: Figure S1). Conversely, serum 25(OH)D concentrations significantly increased $(\approx 70 \%$, range $=7-175 \%)$ in the vitamin $D$ group at 168 -h $(47.9(7.6) \mathrm{ng} / \mathrm{mL})$. The $\%$ increase in serum 25(OH)D concentrations from Bsl to 168-h inversely correlated with Bsl serum 25(OH)D concentrations in the supplemental vitamin D group (Figure 1), which confirms and extends those reported previously [22].

Damaging-exercise modulates circulating calcium and PTH concentrations. The increase in plasma calcium $(\approx 4 \%)$ concentrations at Post and the decrease $(\approx 26 \%)$ in PTH concentrations at 1-h (Figure 2) were not modulated 
Table 1 Baseline (BsI) subject characteristics

\begin{tabular}{lcc}
\hline & Placebo & Vitamin D \\
\hline $\mathrm{n}$ & 13 & 15 \\
Age $(\mathrm{y})$ & $31(5)$ & $30(6)$ \\
Height $(\mathrm{cm})$ & $180(6)$ & $176(7)$ \\
Body mass $(\mathrm{kg})$ & $87.4(14.0)$ & $85.3(17.0)$ \\
BMl $(\mathrm{kg} / \mathrm{m} 2)$ & $26.9(4.2)$ & $27.6(5.6)$ \\
25(OH)D $(\mathrm{ng} / \mathrm{mL})$ & $31.0(8.2)$ & $30.5(9.4)$ \\
Sodium $(\mathrm{mmol} / \mathrm{L})$ & $140(3)$ & $142(2)$ \\
Potassium $(\mathrm{mmol} / \mathrm{L})$ & $4.28(0.31)$ & $4.17(0.15)$ \\
Chloride $(\mathrm{mmol} / \mathrm{L})$ & $104(2)$ & $105(2)$ \\
CO2 $(\mathrm{mmol} / \mathrm{L})$ & $28.0(2.7)$ & $27.7(2.0)$ \\
Glucose $(\mathrm{mg} / \mathrm{dL})$ & $87.1(13.8)$ & $86.9(10.5)$ \\
BUN $(\mathrm{mg} / \mathrm{dL})$ & $16.8(4.1)$ & $14.7(3.0)$ \\
Creatinine $(\mathrm{mg} / \mathrm{dL})$ & $1.00(0.11)$ & $1.08(0.12)$ \\
Total protein $(\mathrm{g} / \mathrm{dL})$ & $7.65(0.33)$ & $7.87(0.53)$ \\
Albumin $(\mathrm{g} / \mathrm{dL})$ & $4.42(0.24)$ & $4.64(0.18)$ \\
Total bilirubin $(\mathrm{mg} / \mathrm{dL})$ & $0.75(0.32)$ & $0.96(0.48)$ \\
\hline
\end{tabular}

Data presented as mean (SD).

27 subjects were Caucasian.

1 subject was non-Hispanic black (Placebo).

by supplemental vitamin D. Although supplemental vitamin D did not significantly moderate calcium or PTH changes, there were, however, fluctuations in calcium and PTH. Plasma calcium concentrations increased above the clinical reference range $(8.2$ to $10.4 \mathrm{mg} / \mathrm{dL}$ ) in four-supplemental vitamin D subjects after the exercise protocol (at Post, 1-h, 48-h, or 168-h). Plasma PTH concentrations increased above the reference range (15 to $75 \mathrm{pg} / \mathrm{mL}$ ) in oneplacebo subject (at 24-h) and in two-supplemental vitamin D subjects (at Pre, Post, 24-h, 72-h, or 168-h). One

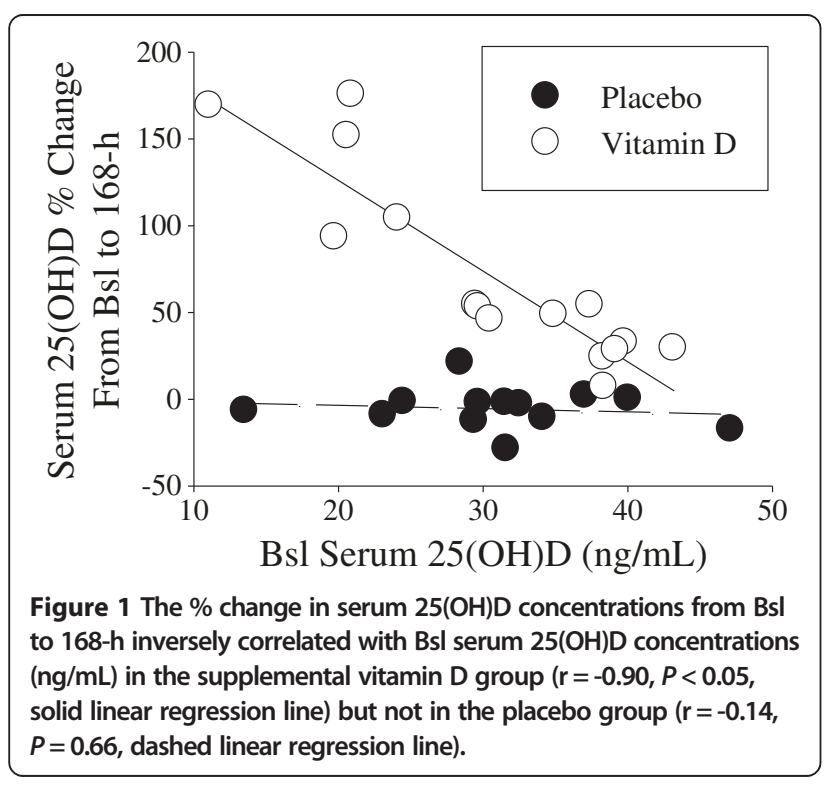

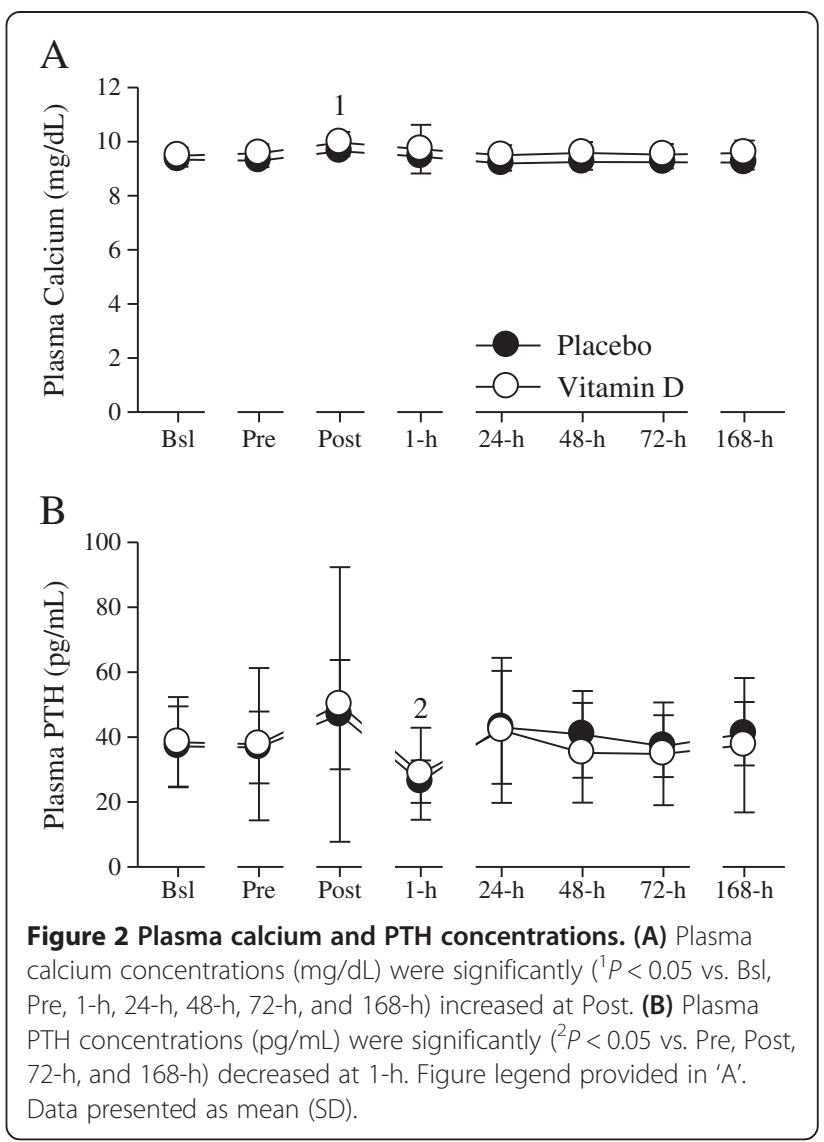

subject in the supplemental vitamin D group displayed a plasma PTH concentration below the clinical reference range at 1 -h.

Single-leg peak isometric force and peak power output. As illustrated in Figure 3, peak isometric force was decreased $(\approx 6 \%)$ in the CON leg immediately and the first 48 -h after the damaging event. The peak isometric force deficits in the SSC leg, however, were more severe $(\approx 32 \%$ at Post, $17 \%$ at $24-\mathrm{h}, 21 \%$ at 48 -h, and $14 \%$ at $72 \mathrm{~h}$ ) and persisted for a longer duration than those in the CON leg. Compared to the $\mathrm{CON}$ leg, peak isometric force was significantly decreased in the SSC leg at Post $(\approx 28 \%)$ and 48 -h $(\approx 17 \%)$. Peak isometric force in the CON and SSC legs were not significantly different between the placebo and vitamin $\mathrm{D}$ groups.

Similar to peak isometric force, the peak power outputdeficits in the SSC leg were more pronounced $(\approx 43 \%$ at Post, $12 \%$ at 24 -h, and $12 \%$ at 48 -h) and persisted for a longer duration than those in the CON leg $(\approx 4$ and $5 \%$ at Post and 24-h, respectively; Figure 4). Peak power output in the SSC leg was significantly decreased $(\approx 39 \%)$ compared to the CON leg at Post. Peak power outputs in the $\mathrm{CON}$ and SSC leg were not significantly different between the placebo and vitamin $\mathrm{D}$ groups. 


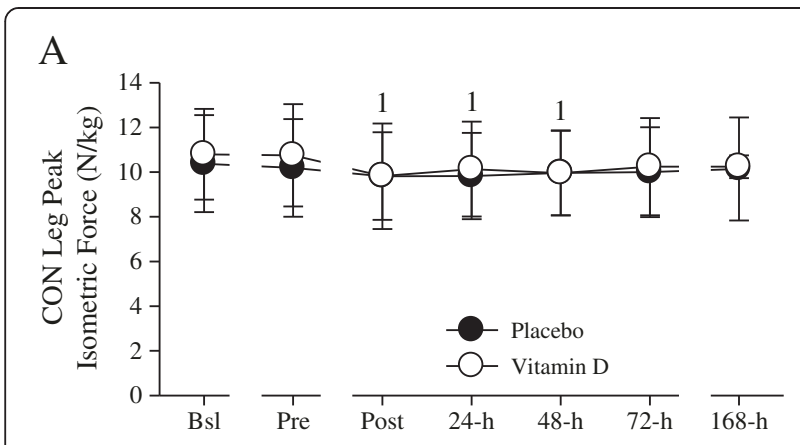

B

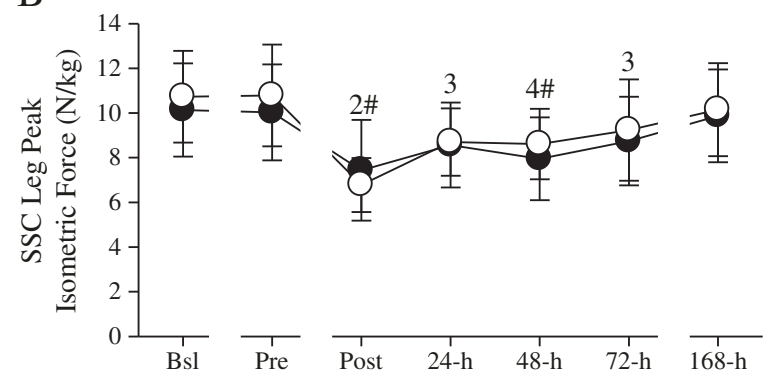

Figure 3 Single-leg peak isometric force (N/kg). CON- (A) and SSC(B) leg peak isometric forces were not significantly different between the placebo and supplemental vitamin D groups. Peak isometric force significantly decreased in the CON leg at Post, 24-h, and $48-h\left({ }^{1} P<0.05\right.$ vs. BsI and Pre). In the SSC leg, peak isometric force was significantly decreased at Post ( ${ }^{2} P<0.05$ vs. Bsl, Pre, 24-h, 48-h, 72-h, 168-h), 24-h $\left({ }^{3} P<0.05\right.$ vs. Bsl, Pre, and 168-h), 48-h ( ${ }^{4} P<0.05$ vs. Bsl, Pre, 72-h, and 168-h), and 72-h ( ${ }^{3} P<0.05$ vs. Bsl, Pre, and 168-h). Peak isometric force was significantly ( ${ }^{\#} P<0.05$ ) different between the CON and SSC legs at Post and 48-h. Figure legend provided in 'A'. Data presented as mean (SD).

A planned comparison prior to data collection was to normalize the deficits in the SSC leg to the CON leg. Considering the decrements in the CON leg, however, this comparison would underestimate the deficit in the SSC leg induced by the exercise protocol. Therefore, we examined the recovery in peak isometric force and peak power output in the SSC leg and between groups at each time interval after the exercise protocol. The recovery of peak isometric force from Post to 24-h in the SSC leg was significantly greater $(\approx 8 \%)$ in the supplemental vitamin D group (Figure $5 \mathrm{~A}$ ). There was a similar trend $(P=0.10)$ for a greater $(\approx 9 \%)$ recovery of peak power output in SSC leg from Post to 24-h in the supplemental vitamin D group as well (Figure $5 \mathrm{~B}$ ).

Circulating biomarkers of muscle damage. The persistent deficits in peak isometric force and peak power output after the exercise protocol provide evidence of skeletal muscle damage $[23,24]$. Skeletal muscle damage is also identified by increases in blood proteins, such as ALT and AST $[23,25]$. In this investigation, supplemental vitamin D blunted the ALT and AST increases after

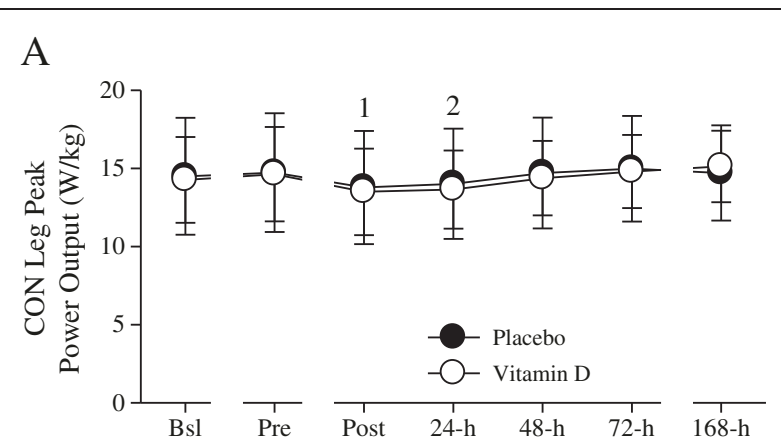

B

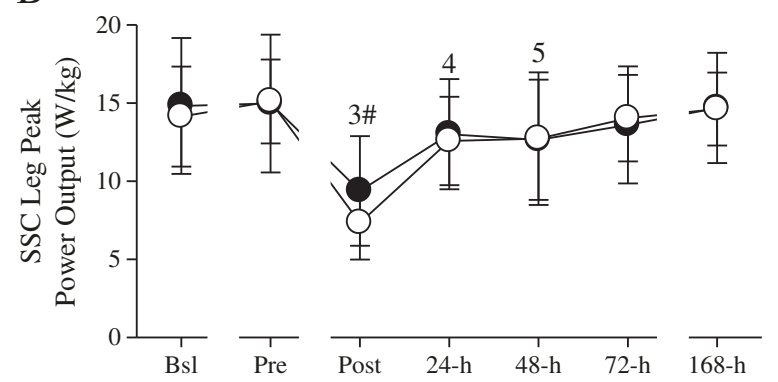

Figure 4 Single-leg peak power output (W/kg). CON- (A) and SSC- (B) leg peak power outputs were not significantly different between the placebo and supplemental vitamin D groups. Peak power output was significantly decreased at Post ( ${ }^{1} P<0.05$ vs. Pre, $24-h, 48-h$, and $\left.72-h\right)$ and $24-h\left({ }^{2} P<0.05\right.$ vs. 48-h and 72-h) in the CON leg. In the SSC leg, peak power output was significantly decreased at Post ${ }^{3} P<0.05$ vs. Bsl, Pre, 24-h, 48-h, 72-h, and 168-h), 24-h ( ${ }^{4} P<0.05$ vs. Bsl, Pre, 72-h, and 168-h), and 48-h ( ${ }^{5} P<0.05$ vs. Pre and 168-h). Peak power output was significantly ( $\left.{ }^{\#} P<0.05\right)$ different between the CON and SSC legs at Post. Figure legend provided in 'A'. Data presented as mean (SD).

(i.e., at 48-h, 72-h, and 168-h) the damaging event (Figure 6A and $\mathrm{B}$ ).

Delayed onset of muscle soreness. Muscle soreness increased immediately and persisted for 24-h to 72-h after the damaging event in the SSC leg (Figure 7A-H). Supplemental vitamin D was ineffective at abrogating muscle soreness in the SSC leg.

\section{Discussion}

The intense exercise protocol induced muscular weakness that persisted for several days (2- to 3-d), which is indicative of skeletal muscle damage [for review see 23]. The recovery in peak isometric force shortly after the intense exercise protocol was enhanced with supplemental vitamin D. Supplemental vitamin D also attenuated the increase in circulating biomarkers representative of muscle damage after the exercise protocol. These novel findings provide evidence that supplemental vitamin $\mathrm{D}$ may serve as an attractive complementary approach to enhance strength recovery shortly after unaccustomed or repetitive contractions that induce persistent muscular weakness in humans.

The enhanced recovery in peak isometric force shortly after (i.e., Post to 24-h) intense exercise suggests that 


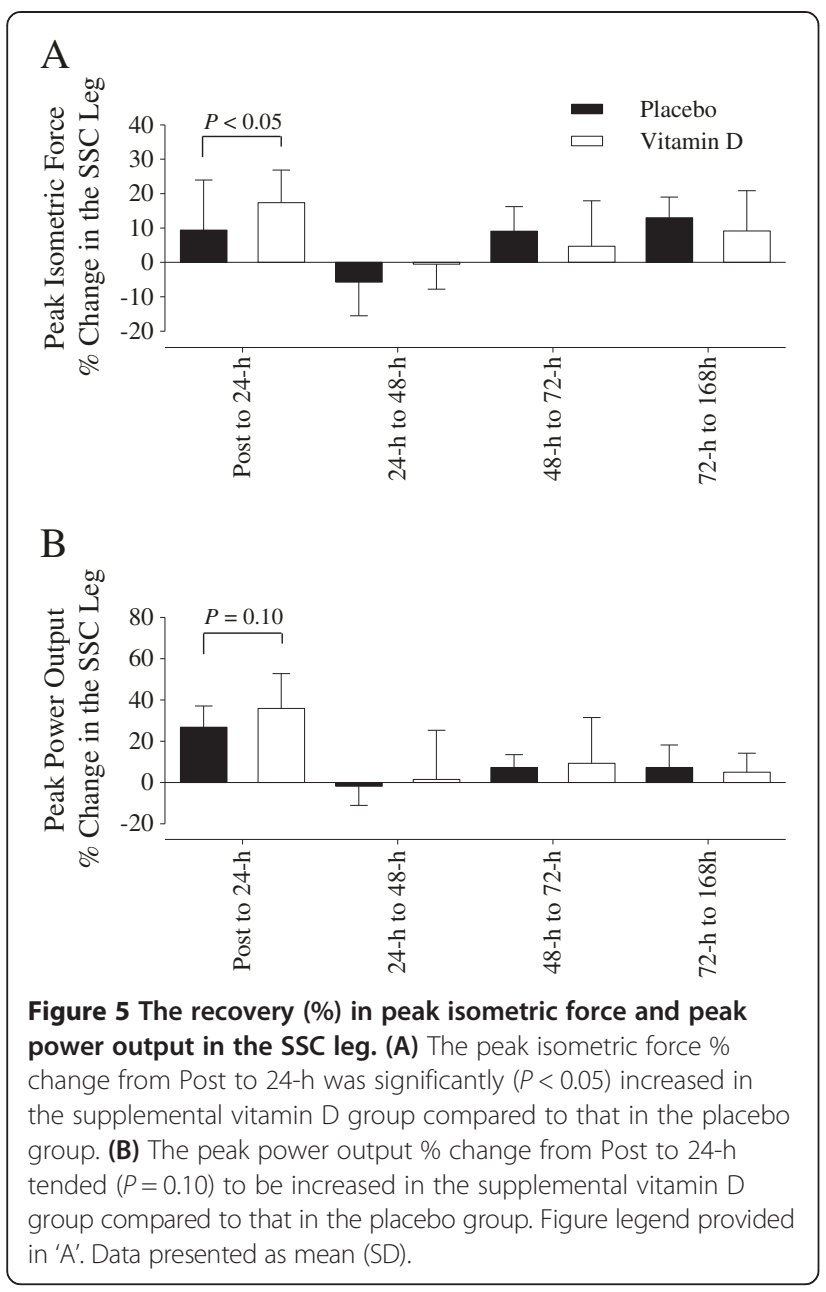

vitamin $\mathrm{D}$ is modulating mechanisms of muscle weakness. A compelling study conducted in severely vitamin Ddeficient (i.e., serum $25(\mathrm{OH}) \mathrm{D}<6.0 \mathrm{ng} / \mathrm{mL}$ ( or $15 \mathrm{nmol} / \mathrm{L}$ )) subjects illustrated recently that vitamin $\mathrm{D}$ treatment (20,000 IU on alternate days for 10-12 weeks) increased serum $25(\mathrm{OH}) \mathrm{D}$ concentrations and reduced the phosphocreatine recovery half-time [26]. Thus, in theory, vitamin $D$ could enhance strength recovery after intense exercise by altering phosphate metabolism or accumulation in skeletal muscle [4,27]. Despite the faster shortterm recovery, vitamin $\mathrm{D}$ did not enhance the delayed (i.e., 24- to 72-h) recovery in peak isometric force after the intense exercise protocol. Therefore, it is plausible that vitamin $\mathrm{D}$ is modulating mechanisms of muscle weakness-induced by fatigue as opposed to muscle damage.

Among other measurement tools, muscle damage is commonly determined by an increase in circulating creatine kinase $(\mathrm{CK})$ or a persistent deficit in skeletal muscle force production $[23,24]$. In experimental rats, vitamin D attenuated the increase in plasma CK activity following intense exercise training [28] and improved force production following a crush injury [15]. Therefore, vitamin
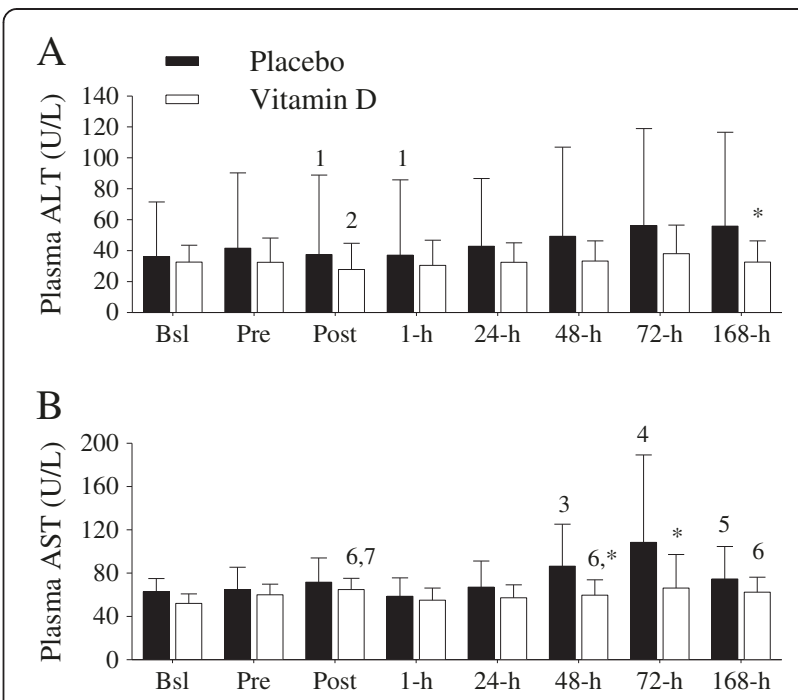

Figure 6 Plasma ALT (U/L) and AST (U/L). (A) Plasma ALT increased ( ${ }^{1} P<0.05$ vs. 24-h, 48-h, 72-h, and 168-h) in the placebo group days after the exercise protocol compared to those at Post and 1-h. Plasma ALT was significantly $\left({ }^{2} P<0.05\right.$ vs. Bsl, Pre, 24-h, 48-h, and 72-h) decreased at Post in the supplemental vitamin D group. Supplemental vitamin D significantly ( $\left.{ }^{*} P<0.05\right)$ decreased ALT compared to the placebo group at 168-h. (B) Plasma AST significantly $\left({ }^{3} P<0.05,48-h\right.$ vs. Bsl and $1-h ;{ }^{4} P<0.05,72-h$ vs. Bsl, Pre, $1-h r$, and $24-h r ;{ }^{5} p<0.05,168-h$ vs. $\left.1-h\right)$ increased in the placebo group during the days following the exercise protocol. In the supplemental vitamin D group, plasma AST was significantly increased at Post $\left({ }^{6} P<0.05\right.$, vs. Bsl; ${ }^{7} P<0.05$ vs. 1 -h), 48-h ( ${ }^{6} P<0.05$, vs. Bsl), and 168-h ( ${ }^{6} P<0.05$, vs. Bsl). Supplemental vitamin D significantly $\left({ }^{*} P<0.05\right)$ attenuated the AST increase at 48-h and 72-h compared to those in the placebo group. Figure legend provided in ' $A$ '. Data presented as mean (SD).

D treatment attenuated the increase in systemic and local biomarkers of muscle damage in experimental rats.

In addition to augmenting strength recovery, supplemental vitamin D ameliorated the increase in plasma ALT and AST after the exercise protocol. The circulating measurements of ALT and AST are used clinically to diagnosis liver damage or failure. ALT and AST are also found in skeletal muscle and released into the circulation after acute muscular damage $[29,30]$. Thus, supplemental vitamin $\mathrm{D}$ could abrogate muscle damage. Instead of inhibiting release from skeletal muscle into the circulation, however, it is plausible that supplemental vitamin D is modulating ALT and AST through an alternative pathway. Clearly future research is needed to confirm the findings from this study, but it is noteworthy that supplemental vitamin D blunted the increase in circulating enzymes representative of skeletal muscle damage after enhancing strength recovery.

Although it is beyond the scope of the present investigation, there are several mechanisms that could explain the enhanced recovery in peak isometric force with supplemental vitamin D. First, mitochondrial oxidative phosphorylation reportedly increases with vitamin D 

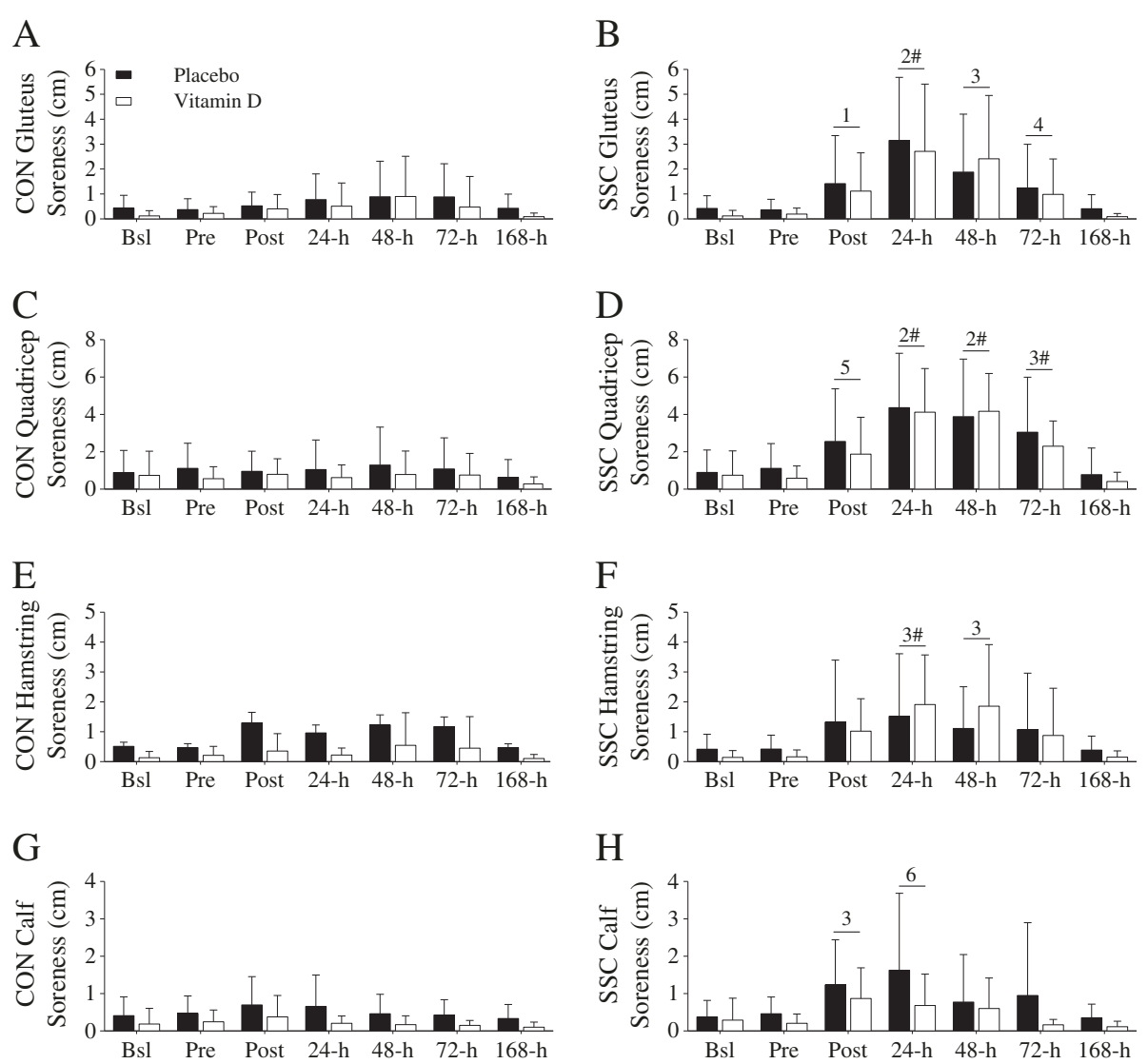

Figure 7 Perceived muscle soreness $(\mathbf{c m})$. Perceived soreness in the gluteus, quadriceps, hamstrings, or calves were not significantly different between the placebo and supplemental vitamin D groups. (A-B) Gluteus soreness increased in the SSC leg at Post ( ${ }^{1} P<0.05$ vs. 24-h and 168-h), 24-h $\left({ }^{2} P<0.05\right.$ vs. Bsl, Pre, 72-h, and 168-h), 48-h ( ${ }^{3} P<0.05$ vs. Bsl, Pre, and 168-h), and 72-h ( ${ }^{4} P<0.05$ vs. 168-h). Gluteus soreness was significantly $\left({ }^{\#} P<0.05\right)$ different between the CON and SSC leg at 24-h. (C-D) Quadriceps soreness increased in the SSC leg at Post ${ }^{5} P<0.05$ vs. Pre, $24-h$, 48-h, and 168-h), 24-h ( ${ }^{2} P<0.05$ vs. Bsl, Pre, 72-h, and 168-h), 48-h ( ${ }^{2} P<0.05$ vs. Bsl, Pre, 72-h, and 168-h), and $72-h\left({ }^{3} P<0.05\right.$ vs. Bsl, Pre, and 168-h). Quadriceps soreness was significantly $(\# P<0.05)$ different between the CON and SSC leg at 24-h, 48-h, and $72-h$. (E-F) Hamstring soreness increased at $24-h\left({ }^{3} P<0.05\right.$ vs. Bsl, Pre, and $\left.168-h\right)$ and $48-h\left({ }^{3} P<0.05\right.$ vs. Bsl, Pre, and $\left.168-h\right)$. Hamstring soreness was significantly $\left({ }^{\#} P<0.05\right)$ different between the CON and SSC leg at 24-h. (G-H) Calf soreness increased at Post $\left({ }^{3} P<0.05\right.$ vs. Bsl, Pre, and 168-h) and 24-h $\left({ }^{6} P<0.05\right.$ vs. $72-h$ and $\left.168-h\right)$. Calf soreness was not significantly different between the CON and SSC legs. Figure legend provided in ' $A$ '. Data presented as mean (SD).

treatment in severely vitamin D-deficient adults [26], which in theory could enhance recovery. Second, supplemental vitamin D could enhance strength recovery by inhibiting apoptosis and increasing extracellular matrix proteins [15]. Third, supplemental vitamin D increases serum $25(\mathrm{OH}) \mathrm{D}$ concentrations, which consequentially, could increase the vitamin D receptor (VDR) in skeletal muscle $[28,31]$. The increased expression of the VDR could impact muscle regeneration and function as it regulates protein synthesis [32] and calcium transport [6]. However, the existence of the VDR in skeletal muscle is controversial [33] and future research is needed to confirm the influence of increasing serum 25(OH)D concentrations on the expression of the VDR in skeletal muscle. Finally, CYP27B1 increases in regenerating skeletal muscle [11]. The increase in CYP27B1 could increase the conversion of $25(\mathrm{OH}) \mathrm{D}$ to $1,25(\mathrm{OH}) \mathrm{D}$, which is the predominately-active metabolite form of vitamin $\mathrm{D}$ that could aid in muscle regeneration. Thus, there are several putative mechanisms that vitamin $\mathrm{D}$ could modulate to enhance strength recovery after intense exercise.

Supplemental vitamin D at $4000 \mathrm{IU} / \mathrm{d}$ does not modulate plasma calcium [34] but significantly decreases plasma PTH [35] in adults with initially low serum $25(\mathrm{OH}) \mathrm{D}$ concentrations (i.e., $<20 \mathrm{ng} / \mathrm{mL}$ ). In this study consisting of subjects with mostly sufficient serum $25(\mathrm{OH}) \mathrm{D}$ concentrations prior to supplementation, supplemental vitamin D did not significantly influence plasma calcium or PTH concentrations. It is noteworthy, however, that one subject displayed a decrease in PTH below its reference range and four subjects displayed increases in plasma calcium above its reference range after the exercise protocol in supplemental vitamin D group. Since these observations were not found prior to exercise, it is likely that the decrease in 
PTH and increase in calcium were mediated by physical exertion as opposed to supplemental vitamin D. Conversely, PTH concentrations increased above its reference range before and after the exercise protocol in two-supplemental vitamin D subjects. The PTH increases occurred in the absence of concomitant fluctuations in plasma calcium above or below its reference range. Therefore, the physiological impact of PTH increases following daily supplemental vitamin D at 4000 IU is unclear. Given the inter-regulatory actions [36-39], future research addressing the influence of supplemental vitamin D on PTH and calcium prior to and following intense exercise is warranted. Future studies are also encouraged to consider possible plasma volume, magnesium level, and ionized calcium changes when investigating the role of vitamin $\mathrm{D}$ on PTH fluctuations prior to and after intense exercise.

There are a couple of limitations of the exercise protocol that should be discussed. First, some subjects performed the exercise protocol until volitional exhaustion while others completed the protocol. Thus, there are likely differences in exercise volume between subjects. Along these lines, there was a tendency for the vitamin D group to perform less jumps but more presses, although these differences were not significant between groups. Second, the vitamin D group tended to display greater decreases in peak isometric forces and peak power outputs at Post. This could suggest greater fatigue after the exercise protocol and a greater capacity for a faster recovery. Future studies are needed to confirm the present findings and would benefit from a controlled exercise volume.

As alluded to above, a limitation to this study is that it does not identify if supplemental vitamin D is modulating fatigue- or damage-related mechanisms, or both, to enhance strength recovery after the exercise protocol. Considering that vitamin $\mathrm{D}$ enhanced recovery the first 24-h and not thereafter suggests that vitamin D might be altering fatigue as opposed to damage-related mechanisms of weakness. Additionally, muscle biopsies were not obtained to ascertain microscopic alterations in skeletal muscle indicative of damage. Another limitation of this study is that subjects were not block randomized according to vitamin D status prior to supplementation. Also, this study was conducted in a modest number of reportedly healthy and active subjects, who for the most part, were vitamin D sufficient prior to supplementation. We speculate that the good vitamin D levels are the result of healthy lifestyles and dietary habits since none of the subjects were supplementing.

\section{Conclusions}

Muscular weakness induced by intense or unaccustomed movements is a major impairment that hinders millions of people worldwide every year. We provide new data demonstrating that supplemental vitamin D could enhance the recovery of force production after intense exercise that mediates a persistent deficit in muscular strength. Supplemental vitamin D also attenuated the increase in circulating biomarkers representative of muscle damage. These findings were observed in subjects with good vitamin D levels, for the most part, and who were modestly active and reportedly healthy prior to supplementation. Based on these findings, we conclude that supplemental vitamin D may serve as an attractive complementary approach to enhance the recovery of skeletal muscle strength following intense exercise in subjects who are vitamin D sufficient and physically active prior to supplementation.

\section{Additional file}

Additional file 1: Figure S1. Serum $25(\mathrm{OH}) \mathrm{D}$ concentrations ( $\mathrm{ng} / \mathrm{mL}$ ). Serum $25(\mathrm{OH}) \mathrm{D}$ concentrations increased in the placebo $\left({ }^{1} P<0.05 \mathrm{vs}\right.$. Bsl, Pre, 1-h, 48-h, 72-h, and 168-h) and supplemental vitamin $D\left({ }^{2} P<0.05\right.$ vs. Pre and $72-h)$ groups at Post. Supplemental vitamin D increased serum $25(\mathrm{OH}) \mathrm{D}$ concentrations ( ${ }^{3} P<0.05$ vs. Bsl; ${ }^{*} P<0.05$ vs. corresponding Placebo). Data presented as mean (SD).

\section{Abbreviations}

ALT: Alanine aminotransferase; AST: Aspartate aminotransferase; CON: Control leg; PTH: Parathyroid hormone; SSC: Exercise leg; 25(OH)D: 25-hydroxyvitamin D.

Competing interests

ES and BD are employed by USANA Health Sciences, Inc

\section{Author contributions}

$\mathrm{TB}, \mathrm{BD}$, and LW contributed to the conception and design of the experiments. $\mathrm{TB}, \mathrm{ES}, \mathrm{BD}, \mathrm{VH}$, and $\mathrm{LW}$ contributed to the collection, analysis, and/or interpretation of the data. TB drafted the article, and TB, ES, BD, VH, and $L W$ revised it critically for important intellectual content. All the authors approved the final version of the manuscript and qualify for authorship, and all of those who qualify for authorship are listed.

\section{Acknowledgements}

We would like to thank Penny Snow, Kristi Thunell, and Michelle (Shelly) Oliver (The Orthopedic Specialty Hospital) for the phlebotomy; the late Adam S. Dern (USANA Health Sciences, Inc.) for the serum sample preparations; and all the subjects that participated in this study. This work was funded in part by the Intermountain Research and Medical Foundation (Salt Lake City, UT, USA) (TB).

\section{Author details}

${ }^{1}$ The Orthopedic Specialty Hospital, 5848 S. Fashion Blvd., Murray, UT 84107, USA. ${ }^{2}$ USANA Health Sciences, Inc, Salt Lake City, UT 84120, USA. ${ }^{3}$ Hyperbaric Medicine, Intermountain Medical Center, Murray, UT, USA 84107 and LDS Hospital, Salt Lake City, UT 84143, USA. ${ }^{4}$ University of Utah, School of Medicine, Salt Lake City, UT 84132, USA.

Received: 26 August 2013 Accepted: 2 December 2013 Published: 6 December 2013

\section{References}

1. Prineas JW, Mason AS, Henson RA: Myopathy in metabolic bone disease. Br Med J 1965, 1:1034-1036.

2. Floyd M, Ayyar DR, Barwick DD, Hudgson P, Weightman D: Myopathy in chronic renal failure. Q J Med 1974, 43:509-524.

3. Irani PF: Electromyography in nutritional osteomalacic myopathy. J Neurol Neurosurg Psychiatry 1976, 39:686-693. 
4. Birge SJ, Haddad JG: 25-hydroxycholecalciferol stimulation of muscle metabolism. J Clin Invest 1975, 56:1100-1107.

5. de Boland $A R$, Boland $R$ : In vitro cellular muscle calcium metabolism. Characterization of effects of 1,25-dihydroxy-vitamin D3 and 25-hydroxyvitamin D3. Z Naturforsch C 1985, 40:102-108.

6. de Boland AR, Boland RL: Rapid changes in skeletal muscle calcium uptake induced in vitro by 1,25-dihydroxyvitamin D3 are suppressed by calcium channel blockers. Endocrinology 1987, 120:1858-1864.

7. Boland R, de Boland AR, Marinissen MJ, Santillan G, Vazquez G, Zanello S: Avian muscle cells as targets for the secosteroid hormone 1,25-dihydroxyvitamin D3. Mol Cell Endocrinol 1995, 114:1-8.

8. Morelli S, Buitrago C, Vazquez G, de Boland AR, Boland R: Involvement of tyrosine kinase activity in 1alpha,25(OH)2-vitamin D3 signal transduction in skeletal muscle cells. J Biol Chem 2000, 275:36021-36028.

9. Buitrago C, Boland R, de Boland AR: The tyrosine kinase $\mathrm{c}-\mathrm{Src}$ is required for 1,25(OH)2-vitamin D3 signalling to the nucleus in muscle cells. Biochim Biophys Acta 2001, 1541:179-187.

10. Buitrago CG, Arango NS, Boland RL: 1alpha,25(OH)2D3-dependent modulation of Akt in proliferating and differentiating $\mathrm{C} 2 \mathrm{C} 12$ skeletal muscle cells. J Cell Biochem 2012, 113:1170-1181.

11. Srikuea R, Zhang X, Park-Sarge OK, Esser KA: VDR and CYP27B1 are expressed in $\mathrm{C} 2 \mathrm{C} 12$ cells and regenerating skeletal muscle: potential role in suppression of myoblast proliferation. Am J Physiol Cell Physiol 2012, 303:C396-C405.

12. Ceglia L: Vitamin D and skeletal muscle tissue and function. Mol Aspects Med 2008, 29:407-414.

13. Boland RL: VDR activation of intracellular signaling pathways in skeletal muscle. Mol Cell Endocrinol 2011, 347:11-16

14. Barker T, Henriksen VT, Martins TB, Hill HR, Kjeldsberg CR, Schneider ED, Dixon BM, Weaver LK: Higher serum 25-hydroxyvitamin D concentrations associate with a faster recovery of skeletal muscle strength after muscular injury. Nutrients 2013, 5:1253-1275.

15. Stratos I, Li Z, Herlyn P, Rotter R, Behrendt AK, Mittlmeier T, Vollmar B: Vitamin $D$ increases cellular turnover and functionally restores the skeletal muscle after crush injury in rats. Am J Pathol 2012, 182:895-904.

16. Barker T, Martins TB, Hill HR, Kjeldsberg CR, Trawick RH, Weaver LK, Traber MG Low vitamin $D$ impairs strength recovery after anterior cruciate ligament surgery. J Evidence-Based Compl Altern Med 2011, 16:201-209.

17. Barker T, Henriksen VT, Martins TB, Kjeldsberg CR, Hill HR: Fluctuations in the skeletal muscle power-velocity relationship and interferon-gamma after a muscle-damaging event in humans. Extreme Physiol Med 2012, 1:1-6.

18. Barker T, Martins TB, Hill HR, Kjeldsberg CR, Henriksen VT, Dixon BM, Schneider ED, Dern A, Weaver LK: Different doses of supplemental vitamin D maintain interleukin-5 without altering skeletal muscle strength: a randomized, double-blind, placebo-controlled study in vitamin D sufficient adults. Nutr Metab (Lond) 2012, 9:16.

19. Barker T, Leonard SW, Hansen J, Trawick RH, Ingram R, Burdett G, Lebold KM, Walker JA, Traber MG: Vitamin E and C supplementation does not ameliorate muscle dysfunction following anterior cruciate ligament surgery. Free Radic Biol Med 2009, 47:1611-1618.

20. Givoni NJ, Pham T, Allen TJ, Proske U: The effect of quadriceps muscle fatigue on position matching at the knee. J Physio/ 2007, 584:111-119.

21. Holick MF, Binkley NC, Bischoff-Ferrari HA, Gordon CM, Hanley DA, Heaney RP, Murad $\mathrm{MH}$, Weaver CM: Evaluation, treatment, and prevention of vitamin D deficiency: an endocrine society clinical practice guideline. J Clin Endocrinol Metab 2011, 96:1911-1930.

22. Close GL, Leckey J, Patterson M, Bradley W, Owens DJ, Fraser WD, Morton JP: The effects of vitamin $\mathrm{D}(3)$ supplementation on serum total 25[OH]D concentration and physical performance: a randomised dose-response study. Br J Sports Med 2013, 47:692-696.

23. Warren GL, Lowe DA, Armstrong RB: Measurement tools used in the study of eccentric contraction-induced injury. Sports Med 1999, 27:43-59.

24. Paulsen G, Mikkelsen UR, Raastad T, Peake JM: Leucocytes, cytokines and satellite cells: what role do they play in muscle damage and regeneration following eccentric exercise? Exerc Immunol Rev 2012, 18:42-97.

25. Sayers SP, Hubal MJ: Histological, chemical, and functional manifestations of muscle damage. In Skeletal Muscle Damage and Repair. Firstth edition. Edited by Tiidus PM. Champaign: Human Kinetics; 2008:37-48.

26. Sinha A, Hollingsworth KG, Ball S, Cheetham T: Improving the vitamin D status of vitamin D deficient adults is associated with improved mitochondrial oxidative function in skeletal muscle. J Clin Endocrinol Metab 2013, 98:E509-E513.
27. de Boland $A R$, Albornoz LE, Boland R: The effect of cholecalciferol in vivo on proteins and lipids of skeletal muscle from rachitic chicks. Calcif Tissue Int 1983, 35:798-805.

28. Choi M, Park H, Cho S, Lee M: Vitamin D supplementation modulates inflammatory responses from the muscle damage induced by highintensity exercise in SD rats. Cytokine 2013, 63:27-35.

29. Nathwani RA, Pais S, Reynolds TB, Kaplowitz N: Serum alanine aminotransferase in skeletal muscle diseases. Hepatology 2005, 41:380-382.

30. Bruunsgaard H, Galbo H, Halkjaer-Kristensen J, Johansen TL, MacLean DA, Pedersen BK: Exercise-induced increase in serum interleukin- 6 in humans is related to muscle damage. J Physiol 1997, 499(Pt 3):833-841.

31. Pojednic RM, Ceglia L, Lichtenstein A, Dawson-Hughes B, Fielding R: Skeletal muscle vitamin $D$ receptor associated with serum 25 -hydroxyvitamin $D$ [abstract]. FASEB J 2013, 27:644.3.

32. Sorensen $\mathrm{OH}$, Lund B, Saltin B, Lund B, Andersen RB, Hjorth L, Melsen F, Mosekilde L: Myopathy in bone loss of ageing: improvement by treatment with 1 alpha-hydroxycholecalciferol and calcium. Clin Sci (Lond) 1979, 56:157-161.

33. Wang $Y$, DeLuca HF: Is the vitamin d receptor found in muscle? Endocrinology 2011, 152:354-363.

34. Vieth R, Chan PC, MacFarlane GD: Efficacy and safety of vitamin D3 intake exceeding the lowest observed adverse effect level. Am J Clin Nutr 2001, 73:288-294

35. Vieth R, Kimball S, Hu A, Walfish PG: Randomized comparison of the effects of the vitamin D3 adequate intake versus $100 \mathrm{mcg}$ (4000 IU) per day on biochemical responses and the wellbeing of patients. Nutr J 2004, $3: 8$

36. Garabedian M, Holick MF, DeLuca HF, Boyle IT: Control of 25-hydroxycholecalciferol metabolism by parathyroid glands. Proc Natl Acad Sci U S A 1972, 69:1673-1676.

37. Rasmussen $\mathrm{H}$, Wong M, Bikle D, Goodman DB: Hormonal control of the renal conversion of 25-hydroxycholecalciferol to 1,25-dihydroxycholecalciferol. J Clin Invest 1972, 51:2502-2504.

38. Fraser DR, Kodicek E: Regulation of 25-hydroxycholecalciferol-1-hydroxylase activity in kidney by parathyroid hormone. Nat New Biol 1973, 241:163-166.

39. Boyle IT, Gray RW, DeLuca HF: Regulation by calcium of in vivo synthesis of 1,25-dihydroxycholecalciferol and 21,25-dihydroxycholecalciferol. Proc Natl Acad Sci U S A 1971, 68:2131-2134.

\section{doi:10.1186/1743-7075-10-69}

Cite this article as: Barker et al:: Supplemental vitamin D enhances the recovery in peak isometric force shortly after intense exercise. Nutrition \& Metabolism 2013 10:69.

\section{Submit your next manuscript to BioMed Central and take full advantage of:}

- Convenient online submission

- Thorough peer review

- No space constraints or color figure charges

- Immediate publication on acceptance

- Inclusion in PubMed, CAS, Scopus and Google Scholar

- Research which is freely available for redistribution 